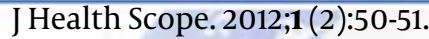 \\ Health Scope \\ www.jhealthscope.com

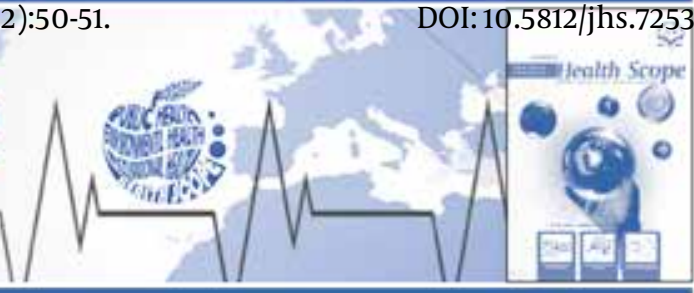

\section{Health Scope in Iran: The Way Forward}

\author{
Abdolvahab Baghbanian ${ }^{1^{*}}$ \\ ${ }^{1}$ Health Promotion Research Centre, Zahedan University of Medical Sciences, Zahedan, IR Iran
}

\begin{tabular}{l}
\hline A R T I C L E I N F O \\
\hline Article type: \\
Editorial \\
\hline Article history: \\
Received: 15 Jul 2012 \\
Revised: 17 Jul 2012 \\
Accepted: 24 Jul 2012 \\
\hline Keywords: \\
Health \\
Policy Making \\
Iran
\end{tabular}

At a time of rapid change in science, technology, demography, epidemiology as well as globalization, global warming and accompanying economic and political movements, public health policy is increasingly becoming complex and inter-dependent. Researchers and policy-makers in all countries are currently facing a series of significant challenges in the funding and delivery of health care. Examples include: the growing burden of chronic diseases, challenges of medical errors, the uptake of advanced and technologically diagnostic and therapeutic procedures, demand pressures, workforce shortages, the scarcity of available resources, the increasing technical complexity of medical equipment and the entrenched interests of the diverse professions running the hospital systems. The health system is further stretched by the rapid ageing of the population and changing disease patterns, the fuzzy boundaries between the public and private sectors, and the increasingly old-fashioned organizations of available health services. Persistent concerns about the quality and safety of health care along with inequalities in health, between the most and the least disadvantaged areas, improves further complexity (1). All Together these challenges and similar problems are inexorably driving
- Implication for health policy/practice/research/medical education:

This editorial reminds healthcare authorities of the importance of research evidence for policy-making. It demonstrates the way journal articles can contribute to knowledge-sharing among health professionals, researchers and decision-makers in order to make wise choices.

- Please cite this paper as:

Baghbanian A. Health Scope in Iran: The Way Forward.J Health Scope. 2012; 1 (2): 50-1.

increased demands for healthcare service, rising costs and complexity, and are already analysising the limits of the financial, physical and human resources necessary to improve the health of communities. They point to the possibility of extensive challenges in developing countries including Islamic Republic (I. R. ) of Iran, and remind us that there would be no room for complacency, or for inertia in reforming their healthcare systems.

Overall, I. R. Iran most of the time provides a fairly good health care to the majority of its citizens, even though it does not compare well with like countries. Their rank is less than the average ranking of Eastern Mediterranean countries in terms of many aspects of health status. For example, it has lower life expectancy (72. 1 years), and higher Infant Mortality Rate (18 deaths per 1000 births) compared with many countries in the region, as of 2010. Total health expenditure, as a proportion of Gross Domestic Product, accounted for 5. 5\%, slightly lower than the Eastern Mediterranean average of 5. 6\% in 2010 (2). Nonetheless, there have been several encouragements to improve health - e.g. the establishment of the healthcare network in 1984, the Urban Inpatient Insurance Scheme

\footnotetext{
* Corresponding author: Abdolvahab Baghbanian. Public Health Department, Faculty of Health, Mashahir Square, Zahedan, Zahedan, IR Iran. Tel/Fax: +98-9173132347, E-mail: abag2253@uni.sydney. edu.au
} 
in 2000 , the endorsement of the family physician law in 2004, and the Rural Health Insurance Scheme in 2005 (2); however, health system remains committed to costly medical advances, labor-intensive rescue and the preservation of traditional values and practices. Considering the current health infrastructure, little flexibility exists to achieve this in a system hamstrung by a focus on historical funding, fee-for-service payment method and isolated episodes of acute care, growing out-of-pocket expenses, workforce deficiencies and inadequate insurance coverage. For instance, it has been estimated that out-of-pocket spending on health is higher relative to many Eastern Mediterranean countries, where it has grown from 50.7 in 2008 to $58.9 \%$ in 2010 (2) and currently, for some people, presents a serious barrier to needed care. Similarly, there is worries about equitable access to health care for some people particularly those without a suitable health insurance (i. e. 10-40\% of the population - depending upon the source and study) $(1,3)$. Undoubtedly, there is a long way to go, if researchers and policy-makers are maintaining and improving the current status of health and wellbeing of Iranian residents. The analysis of evidence that informing policy and practicing is a way forward many researchers have attempted to drive continuous improvement. The Iranian Journal of Health Scope with its focus on the research evidence from environmental health, epidemiology, health economics, health policy, health promotion, healthcare management, occupational health, and similar public health related issues has emerged to create a smarter health and wellness for all communities particularly those experiencing significant health challenges in developing countries. The most important contribution of the journal is its ability to provide information and incentives designed to help health policy-makers create wise choices. It takes a meaningful whole-system perspective, in which health-related issues can be placed in context with many other factors impinging on executives' practices. The journal is informative to all researchers in health-related fields and has the potential to add crucial dimensions to executives' understanding of complex, health policy issues.

A few months ago the first issue of the Journal of Health Scope was published to address some of the above health challenges. The central message was a growing need for policy-makers and health professionals who possess the evidence-derived knowledge required to help prevail today's challenges. However, there is a need to appreciate the challenge that faces researchers and policy-makers in communicating that knowledge. Through effective communication researchers should ensure that their research appropriately informs judgments for which policy-makers are responsible. This challenge can be tackled with a focus on the big picture of the situation of inquiry, national leadership and shared vision. I have faith in nationwide cooperation and leadership is required to establish mean- ingful interactions between researchers and policy-makers in order to secure and improve Iran's future health. When policy-makers confer with researchers, the quality of their policy decisions is enhanced because of the reflective practice and peer support networks that are created through interactions (4-6). A well-organized alternative based on these attributes would help build a better health system that can provide better health outcome and better value for money. There are exciting times for public health academic and practice communities including epidemiologists, environmental and occupational health professionals, and policymakers to demonstrate their utility and rigor in this period of rapid change. Academia and health are generally going through tough times. They should try to do better than the complexities and uncertainties of their environment and personal powers permit. What are needed in such a situation are the use of empirical evidence in decision-making and the involvement of researchers in public health policies. I welcome the opportunity to invite academics, health managers and professionals to contribute to this collaboration, especially on the public health-related topics in developing countries. It is essential that policy-makers (and researchers) gather to tackle the challenges such as all of the above, if they are to maintain and improve the current status of health and wellbeing of their populations. I suggest that the journal of health scope has the potential to contribute to such atmosphere.

\section{Funding/Support}

None declared.

\section{References}

1. Group TWB. Islamic Republic of Iran Health Sector Review. Sector HD. Report No. :39970 - IR. 2007; Available from: http://medolympiad. behdasht.gov. ir/uploads/280_954_First_Modiriat3.pdf.

2. Mediterranean WROftE. Regional Health Observatory: Database. 2012; Available from: http://rho. emro. who. int/rhodata/.

3. Bayat F. Impact of dental insurance on adults' oral health care in Tehran, Iran. Finland: Faculty of Medicine, University of Helsink; 2010; Available from: https://www. doria. fi/bitstream/handle/10024/61764/impactof. pdf.

4. Baghbanian A. The Emergence of Adaptive Decision-making in Complex Health Systems: Economics and Resourcing of Complex Health Systems: The Emergence of Adaptive Decision-making in Health Care. LAP Lambert Acad. Publ. ; 2011.

5. Baghbanian A, Hughes I, Kebriaei A, Khavarpour FA. Adaptive decision-making: how Australian healthcare managers decide. Aust Health Rev. 2012;36 (1):49-56.

6. Baghbanian A, Torkfar G, Baghbanian Y. Decision-Making in Australia's Healthcare System and Insights From Complex Adaptive Systems Theory. J Health Scope. 2012;1 (1):29-38. 